\title{
Colitis isquémica y neumatosis intestinal en paciente con COVID-19: reporte de caso
}

Jorge Luis Vélez Páez ${ }^{1, a, b}$; Christian Leonardo Mora Coello ${ }^{1,2}$; Wilson Daniel Álava Muñoz ${ }^{1,3}$; María José Proaño Constante ${ }^{1,2}$; Milagros Esthela Heredia Toledo ${ }^{1}$; Santiago Xavier Aguayo Moscoso*1,a

\section{RESUMEN}

El coronavirus tipo 2 del síndrome respiratorio agudo grave (SARS-CoV-2) es el virus causante de la pandemia actual. En general, los pacientes infectados con SARS-CoV-2 desarrollan enfermedades respiratorias. Los síntomas iniciales son fiebre, tos y disnea que progresan con rapidez a una neumonía; además, se han reportado afectaciones extrapulmonares como las gastrointestinales.

Está descrito que el receptor de la enzima convertidora de angiotensina 2 (ACE2) y la serina proteasa TMPRSS2 para cebado de la proteína S facilitan el ingreso del SARS-CoV-2 a las células. Estas proteínas se expresan en células alveolares tipo 2 y también en los enterocitos del íleon y colon, por lo que se ha detectado el virus en el tracto digestivo ${ }^{(1)}$.

Presentamos el caso de un paciente con neumonía por COVID-19, que también presentó neumatosis intestinal y colitis isquémica, ambas manifestaciones gastrointestinales.

Palabras clave: SARS CoV-2; COVID-19; Colitis isquémica (Fuente: DeCS BIREME).

\section{Ischemic colitis and pneumatosis intestinalis in a patient with COVID-19: a case report}

\section{ABSTRACT}

The severe acute respiratory syndrome coronavirus 2 (SARS-CoV-2) is the virus causing the ongoing pandemic. In general, patients infected with SARS-CoV-2 develop respiratory diseases. The first symptoms are fever, cough and dyspnea that rapidly progress to pneumonia. Additionally, extrapulmonary manifestations, such as those in the gastrointestinal system, have been reported.

It has been described that the angiotensin-converting enzyme 2 (ACE2) receptor and the transmembrane serine protease 2 (TMPRSS2) for S protein priming favor SARS-CoV-2 entry into the cells. These proteins are expressed in alveolar type II cells as well as in ileum and colon enterocytes, thus detecting the virus in the digestive tract.

We present the case of a patient with COVID-19 pneumonia and gastrointestinal findings such as pneumatosis intestinalis and ischemic colitis.

Keywords: SARS-CoV-2; Coronavirus infections; Colitis, ischemic (Source: MeSH NLM).

\footnotetext{
1 Hospital Pablo Arturo Suárez. Quito, Ecuador.

2 Pontificia Universidad Católica del Ecuador. Quito, Ecuador.

3 Universidad Central del Ecuador. Quito, Ecuador.

a Médico Especialista en Medicina Crítica y Terapia Intensiva.

b Máster en Investigación Clínica y Epidemiología.

*Autor corresponsal.
} 


\section{INTRODUCCIÓN}

La enfermedad por coronavirus 2019 (COVID-19) es una infección altamente contagiosa que tiene un impacto mundial significativo en la morbimortalidad ${ }^{(2)}$.

La clínica gastrointestinal en la COVID-19 se caracteriza por síntomas como náusea, vómito y diarrea. Por otro lado, por medio de la tomografía es posible detectar casos sutiles de neumatosis, hallazgos que se pueden observar en los pacientes con isquemia intestinal antes del desarrollo de necrosis transmural ${ }^{(3)}$. En este contexto, se considera que la neumatosis intestinal es una manifestación de la colitis isquémica, enfermedad relativamente rara que, en el contexto de la infección por SARS-CoV-2 y su alto potencial trombogénico, podría estar asociada con la trombosis in situ (4).

El presente reporte describe el caso clínico de un paciente con neumonía y distrés respiratorio por COVID-19 que desarrolla una colitis isquémica que se evidencia en la tomografía como neumatosis intestinal, cuyas manifestaciones clínicas son inusuales.

\section{REPORTE DE CASO}

Varón de 73 años, con antecedentes de diabetes mellitus tipo 2 (en tratamiento con insulina glargina 45 UI y linagliptina) e hipertensión arterial (telmisartán e hidroclorotiazida $80 / 25 \mathrm{mg}$ ). Además, exposición a tabaco aproximadamente por 30 años (índice paquetes/año o IPA de 30 paquetes-año).

Acudió a consulta por un cuadro clínico de 4 días de evolución, caracterizado por alza térmica, astenia, hiporexia y disnea con episodios de desaturación (SpO2 70 \%), sin reportes de sintomatología gastrointestinal al comenzar las molestias. Al inicio, el paciente requirió hospitalización por 12 días en una institución de salud privada, donde se inició ventilación mecánica, con parámetros ventilatorios elevados con fracción inspirada de oxígeno $\left(\mathrm{FiO}_{2}\right)$ de $100 \%$ y presión positiva al final de la inspiración (PEEP) de 19; valores que descendieron paulatinamente hasta una $\mathrm{FiO}_{2}$ de $45 \%$ y un PEEP de 13. Sufrió un choque distributivo atribuido a sepsis y a los fármacos, para lo cual recibió tratamiento con norepinefrina y dopamina a dosis elevadas.

En el decimotercer día de hospitalización fue transferido a una institución de salud pública para continuar el manejo clínico. Ingresó a la unidad de cuidados intensivos con ventilación mecánica invasiva por la oxigenación severamente comprometida, con la presión arterial de oxígeno/ $/ \mathrm{FiO}_{2}$ $\left(\mathrm{PaFiO}_{2}\right)$ de 93 que requirió ajuste de su ventilación con $\mathrm{FiO}_{2}$ de $90 \%$, PEEP de 6, bajo sedación profunda (RASS -4). La hemodinamia requirió un soporte vasoactivo (norepinefrina). Otros hallazgos fueron los siguientes: piel fría, frecuencia cardiaca de 73 latidos por minuto, llenado capilar de 4 segundos y valor de lactato de 1,3.

Se mantuvo con anticoagulación por presentar valores elevados de dímero $D$ al momento del ingreso $(2647 \mathrm{ng} / \mathrm{ml})$ (Tabla 1$)$. Durante la evolución, el paciente presentó un fallo renal agudo oligúrico con cifras de creatinina mayores de $2 \mathrm{mg} / \mathrm{dl}$, que remitió durante su estadía hospitalaria con el empleo de diuréticos de asa. Asimismo, desarrolló una neumonía grave por COVID-19 complicada con sobreinfección bacteriana pulmonar. Los hemocultivos reportaron crecimiento de cocos grampositivos a las 12 horas, por lo que inició terapia con vancomicina, polimixina $\mathrm{E}$ y amikacina con base en la epidemiología de la unidad.

Tabla1. Análisis de ingreso a la Unidad de Terapia Intensiva

\begin{tabular}{|c|c|c|c|c|c|}
\hline Leucocitos & $15,98 \mathrm{~K} / \mu \mathrm{l}$ & Hematocrito & $46,50 \%$ & Sodio / potasio & $155 / 4,5 \mathrm{meq} / \mathrm{l}$ \\
\hline Neutrófilos & $14,98 \mathrm{~K} / \mu \mathrm{l}$ & AST / ALT & $29 / 34 \mathrm{U} / \mathrm{L}$ & Cloro & $117 \mathrm{meq} / \mathrm{l}$ \\
\hline$(\%)$ & $(89,8 \%)$ & & & & \\
\hline Linfocitos & $0,53 \mathrm{~K} / \mu \mathrm{l}$ & Tiempo de & $11,4 \mathrm{~s}$ & Ferritina & $1231 \mathrm{ng} / \mathrm{ml}$ \\
\hline$(\%)$ & $(3,3 \%)$ & protrombina & & & \\
\hline Monocitos & $0,38 \mathrm{~K} / \mu \mathrm{l}$ & Tiempo de & $26,9 \mathrm{~s}$ & Dímero D & $2647 \mathrm{ng} / \mathrm{ml}$ \\
\hline$(\%)$ & $(2,4 \%)$ & tromboplastina & & & \\
\hline Plaquetas & $285000 \mathrm{~K} / \mu \mathrm{l}$ & INR & 1,08 & Deshidrogenasa láctica & $803 \mathrm{U} / \mathrm{L}$ \\
\hline \multirow[t]{2}{*}{ Hemoglobina } & $16 \mathrm{~g} / \mathrm{dl}$ & Nitrógeno ureico / & $61 / 1,1 \mathrm{mg} / \mathrm{dl}$ & Interleucina 6 & $317 \mathrm{pg} / \mathrm{ml}$ \\
\hline & & Creatinina & & & \\
\hline
\end{tabular}

Durante la valoración clínica rutinaria del abdomen, se evidenció una masa dura en el cuadrante inferior izquierdo de bordes delimitados de $8 \times 6 \mathrm{~cm}$. El paciente no mostró manifestaciones clínicas abdominales ni sangrado digestivo, por lo que se decidió realizar un estudio tomográfico que reportó neumatosis en ángulo cólico derecho, neumatosis mesentérica y una masa palpada que correspondió a un hematoma de pared abdominal de 17,4 ×8,2 x 6,1 cm con un volumen aproximado de $452 \mathrm{~cm} 3$, que se atribuyó a la anticoagulación recibida (Figura 1). 


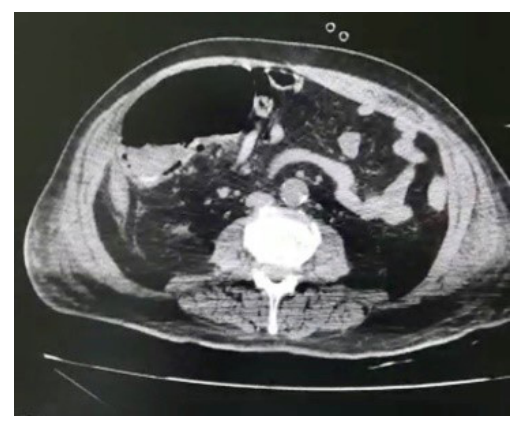

A

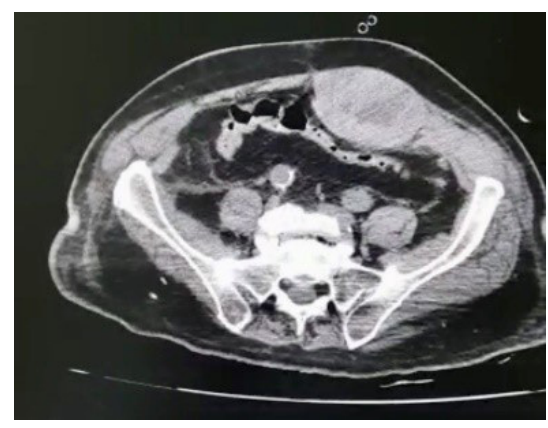

B

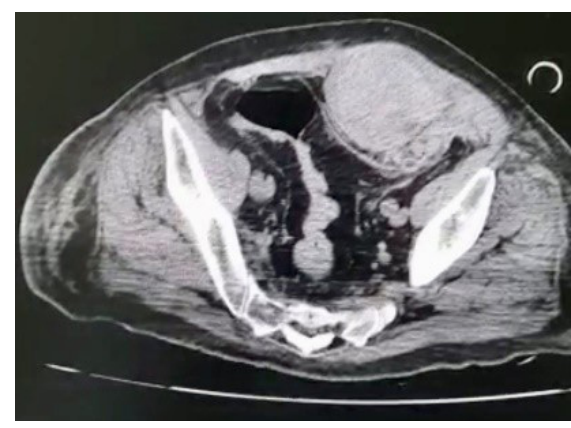

C

Figura 1. TAC simple de abdomen con evidencia de (A) neumatosis en ángulo cólico derecho, $(\mathrm{B})$ neumatosis mesentérica y (C) hematoma abdominal

El paciente recibió manejo clínico ventilatorio invasivo bajo sedoanalgesia y fue valorado por Cirugía General, por lo que se realizó una laparotomía exploratoria ante la probabilidad de que la neumatosis intestinal fuera consecuencia de una

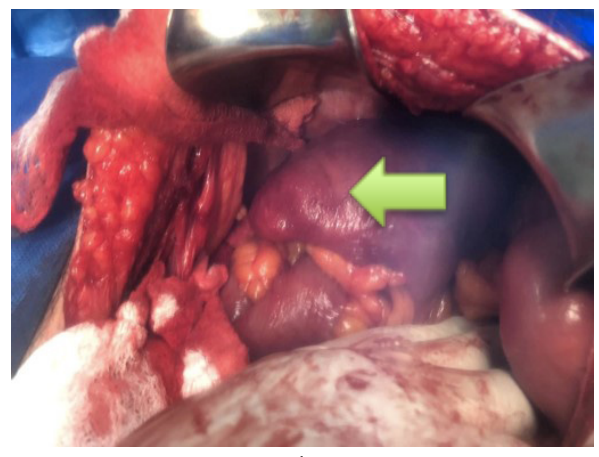

A isquemia mesentérica. Como hallazgo postquirúrgico se reportó una tiflitis y un hematoma de los músculos rectos del abdomen (Figura 2).

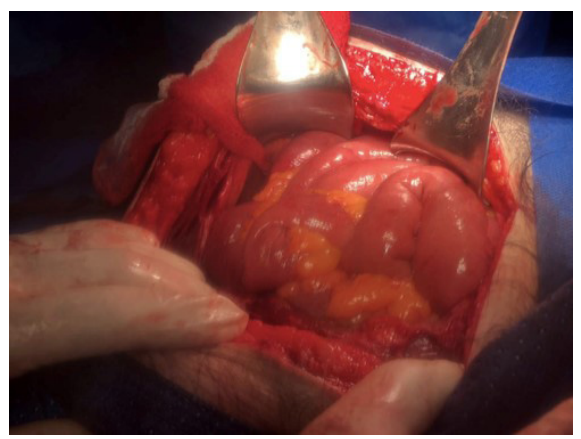

B

Figura 2. Hallazgos quirúrgicos: (A)vista macroscópica cavidad abdominal, con inflamación del ciego (flecha verde) y (B) asas intestinales indemnes

Este paciente tuvo una prolongada estancia hospitalaria en nuestra unidad; después de la cirugía presentó choque refractario y falleció.

\section{DISCUSIÓN}

La COVID-19, ocasionado por el SARS-CoV-2, es una enfermedad de gran diseminación en el mundo. Fue definida como una pandemia en marzo del 2020 y tiene características nosológicas multisistémicas. Se plantea que la principal vía de entrada del virus al organismo es el aparato respiratorio. Sus manifestaciones clínicas son variadas y comprenden afecciones respiratorias, neurológicas, vasculares, así como también gastrointestinales, que aparecen en $16 \%$ de los casos; las más frecuentes son náuseas, vómitos, pérdida de apetito y dolor abdominal; además de manifestaciones específicas hepáticas y pancreáticas ${ }^{(2)}$.

El sistema gastrointestinal tiene una alta expresión de receptores ACE2 ${ }^{(5)}$, por lo que en la COVID-19 destacan las expresiones clínicas hepáticas ${ }^{(6,7)}$ y pancreáticas ${ }^{(8)}$ que son leves y transitorias.

La colitis isquémica es relativamente rara, aunque es la afección isquémica más común del tracto gastrointestinal, y aparece a consecuencia de una hipoperfusión colónica con resultados que pueden ser irreversibles. Existen innumerables causas de isquemia colónica que en un contexto de análisis fisiopatológico pueden definirse como oclusivas y no oclusivas. En jóvenes, la trombofilia y la vasculitis son las causas más importantes entre las oclusivas ${ }^{(4)}$. El paciente tenía factores de riesgo para desarrollo de colitis isquémica: edad, hipertensión y diabetes. Durante su estancia en el hospital desarrolló un choque y falla renal y, además, recibió vasopresores: todos ellos factores asociados a esta entidad. 
En la actualidad, el SARS-CoV-2 se ha relacionado con el desarrollo de coagulopatía y complicaciones tromboembólicas debido a un aumento en los niveles de factor Von-Willebrand, lo que desencadenaría el daño endotelial, activación y trombosis in situ, proceso que es compatible con el desarrollo de afecciones gastrointestinales isquémicas ${ }^{(9)}$.

En nuestro caso, el único hallazgo es la presencia de neumatosis intestinal, pues carecíamos del reporte de un compromiso de flujo en asas intestinales.

La neumatosis intestinal ( $\mathrm{NI}$ ) es rara y de baja incidencia, su presentación clínica puede variar entre cuadros leves y altamente mortales. En el $85 \%$ de los casos es de etiología secundaria y está asociada a enfermedades clínicas severas de fisiopatología autoinmune ${ }^{(10)}$. Du Vernoy la describió por primera vez en 1730 al observar quistes llenos de gas dentro de las capas submucosa y subserosa del intestino en muestras de autopsia ${ }^{(11)}$. Su prevalencia es difícil de determinar porque los pacientes, en su mayoría, son asintomáticos; tiene una tasa de incidencia de aproximadamente $0,03 \%$ entre los 50 a 80 años y predomina en el sexo masculino en una relación 3:1 respecto a las mujeres ${ }^{(12)}$.

En la NI encontramos un hallazgo poco común en los estudios de imágenes: la presencia de gas en la pared intestinal (10) que, en la mayoría de los casos, está localizado en el intestino grueso y, con menor frecuencia, en el intestino delgado. En cualquiera de estas ubicaciones, el gas se encuentra en la capa subserosa o en submucosa intestinal (13).

La neumatosis intestinal puede estar asociada a otros cuadros, por ejemplo, la afección pulmonar, la enfermedad inflamatoria intestinal o la diabetes. También puede ser secundaria al empleo de ciertos medicamentos como los esteroides y agentes de quimioterapia por enfermedad vascular, como en la esclerosis sistémica ${ }^{(13)}$.

Según reportes de casos, la neumatosis intestinal asociada a la COVID-19 es un cuadro poco descrito, que, posiblemente, respondería a la disrupción del "eje intestino-pulmón" (7) asociado a un daño directo de la pared intestinal, con la subsecuente alteración de la microbiota durante la infección por SARS-CoV-2 ${ }^{(6)}$.

Dentro del diagnóstico diferencial, cabe destacar a la tiflitis, que es un proceso inflamatorio que afecta al ciego y al íleon terminal en pacientes neutropénicos y es el resultado de una lesión en la mucosa en un huésped con deterioro del sistema inmunitario. Si analizamos los factores de riesgo de paciente, la diabetes mellitus que cursa con la COVID-19 implica un desequilibrio de la relación proinflamatoriaantiinflamatoria, y que podría exacerbarse por las medidas terapéuticas coadyuvantes (corticoides) en el paciente no inmunodeprimido (se descartó neutropenia). En la NI se ha postulado que la predilección por el íleon terminal y el ciego está relacionada con su grado de distensibilidad y limitación del flujo sanguíneo por su anatomía vascular ${ }^{(14)}$.

Una vez infectados, los enterocitos absorbentes del íleon y del colon podrían destruirse, lo que provocaría manifestaciones de mala absorción, desequilibrio en la secreción intestinal y alteraciones en el sistema nervioso entérico ${ }^{(15)}$. Esta particularidad se observó en nuestro paciente con la intolerancia al aporte nutricional enteral.

Aunque la mayoría de los virus estarían muertos en el ambiente ácido del estómago, todavía existe la posibilidad de que la saliva y las secreciones puedan transportarlo al sistema digestivo, donde la replicación viral puede mantenerse en las células susceptibles ${ }^{(1)}$.

Sin embargo, hasta hoy no se ha dado un consenso directo para el manejo correcto de la NI ${ }^{(16)}$. Por lo tanto, el desafío que tiene el médico clínico incluye la identificación del diagnóstico correcto de $\mathrm{NI}$ frente a otras causas de gas peritoneal, y también debe tomar la decisión de si se requiere una intervención quirúrgica o no. En nuestro caso, el paciente fue intervenido quirúrgicamente y se reportó tiflitis.

Otro parámetro paraclínico significativo para el diagnóstico oportuno es el hallazgo de hiperlactatemia como un factor predictor de $\mathrm{NI}^{(17)}$, característica que no fue evidente durante el desarrollo de la enfermedad.

Dentro de los reportes de tiflitis asociados a COVID-19, existe un caso de desarrollo de esta entidad con un conteo de neutrófilos mayor a $1000 / \mu \mathrm{l}$, que es otra característica única de la enterocolitis inducida por COVID-19 ${ }^{(18)}$. Este paciente no presentó neutropenia al igual que el de nuestro caso.

Cabe indicar que no se realizó biopsia o autopsia, que daría el diagnóstico definitivo, ya que no se consideró necesario.

Durante la pandemia hemos observado que este virus ocasiona afecciones extrapulmonares, en este caso, un compromiso gastrointestinal, lo que nos lleva a considerar que se debe buscar alguna complicación distinta al cuadro respiratorio en un cuadro de COVID-19.

Por ello, concluimos y sugerimos que, dentro del manejo clínico de estos pacientes, es necesario mantener un alto nivel de sospecha de isquemia intestinal en el grupo que tiene los factores de riesgo mencionados y si, además, aparecen características clínicas como dolor abdominal, sangre en las heces y urgencia en la defecación.

\section{REFERENCIAS BIBLIOGRÁFICAS:}

1. Zhang $H$, Kang Z, Gong $H, X u ~ D$, Wang J, Li Z, et al. Digestive system is a potential route of COVID-19: an analysis of single-cell coexpression pattern of key proteins in viral entry process. Gut. 
2020; 69(6): 1010-8.

2. Huang C, Wang Y, Li X, Ren L, Zhao J, Hu Y, et al. Clinical features of patients infected with 2019 novel coronavirus in Wuhan, China. Lancet. 2020; 395(10223): 497-506.

3. Kernagis LY, Levine MS, Jacobs JE. Pneumatosis intestinalis in patientswith ischemia: correlation of CT findings with viability of the bowel. Am J Roentgenol. 2003; 180(3): 733-6.

4. Doulberis M, Panagopoulos P, Scherz S, Dellaporta E, Kouklakis $G$. Update on ischemic colitis: from etiopathology to treatment including patients of intensive care unit. Scand J Gastroenterol. 2016; 51(8): 893-902.

5. Wang $\mathrm{F}$, Wang $\mathrm{H}$, Fan J, Zhang $\mathrm{Y}$, Wang $\mathrm{H}$, Zhao Q. Pancreatic Injury Patterns in Patients with Coronavirus Disease 19 Pneumonia. Gastroenterology. 2020; 159(1): 367-70.

6. Meini S, Zini C, Passaleva MT, Frullini A, Fusco F, Carpi R, et al. Pneumatosis intestinalis in COVID-19. BMJ Open Gastroenterol. 2020; 7(1): e000434.

7. He Y, Wen Q, Yao F, Xu D, Huang Y, Wang J. Gut-lung axis: the microbial contributions and clinical implications. Crit Rev Microbiol. 2017; 43(1): 81-95.

8. Zhao D, Yao F, Wang L, Zheng L, Gao Y, Ye J, et al. A comparative study on the clinical features of COVID-19 pneumonia to other pneumonias. Clin Infect Dis. 2020; ciaa247.

9. Paul T, Joy AR, Alsoub HARS, Parambil JV. Case report: ischemic colitis in severe COVID-19 pneumonia: an unforeseen gastrointestinal complication. Am J Trop Med Hyg. 2021; 104(1): 63-5.

10. Morris MS, Gee AC, Cho SD, Limbaugh K, Underwood S, Ham B, et al. Management and outcome of pneumatosis intestinalis. Am J Surg. 2008; 195(5): 679-82.

11. Pietropaolo MD, Trinci M, Giangregorio C, Galluzzo M, Miele V. Pneumatosis cystoides intestinalis: case report and review of literature. Clin J Gastroenterol. 2020; 13(1): 31-6.

12. Jenkins $M$, Courtney H, Pope E, Williamson J. A case report and approach to management in pneumatosis intestinalis. Ann Med Surg (Lond). 2017; 23: 25-7.

13. Donovan S, Cernigliaro J, Dawson N. Pneumatosis intestinalis: a case report and approach to management. Case Rep Med. 2011; 2011: 571387.

14. Moya-Sánchez E, Fernández-Navarro L, Medina-Salas V. Enterocolitis neutropénica o tiflitis: causa de abdomen agudo en paciente neutropénico. RAPD Online. 2018; 41(3): 159-61.

15. Ettayebi K, Crawford SE, Murakami K, Broughman JR, Karandikar $\mathrm{U}$, Tenge VR, et al. Replication of human noroviruses in stem cellderived human enteroids. Science. 2016; 353(6306): 1387-93.

16. Wayne E, Ough M, Wu A, Liao J, Andresen KJ, Kuehn D, et al. Management algorithm for pneumatosis intestinalis and portal venous gas: treatment and outcome of 88 consecutive cases. J Gastrointest Surg. 2010; 14(3): 437-48.

17. Hawn MT, Canon CL, Lockhart ME, Gonzalez QH, Shore G, Bondora $A$, et al. Serum lactic acid determines the outcomes of CT diagnosis of pneumatosis of the gastrointestinal tract. Am Surg. 2004; 70(1): 19-23.

18. Rehman M, Gondal A, Khan S, Rehman NU, Molina J. Neutropenic enterocolitis and rapid spontaneous resolution of portal venous gas: a non-respiratory manifestation of COVID-19. Cureus. 2020; 12(7): e9445.

\section{Correspondencia:}

Santiago Xavier Aguayo Moscoso

Dirección: Angel Ludeña y Machala 0e52-61, Hospital Pablo Arturo Suárez. Quito, Ecuador.

Teléfono: 593-2-3947-940

Correo electrónico: drsaguayo@gmail.com

\section{Recibido: 13 de septiembre de 2020}

Revisado: 10 de diciembre de 2020 Aprobado: 06 de enero de 2021

( $)$ La revista. Publicado por Universidad de San Martín de Porres, Perú. (cc) Br Licencia de Creative Commons Artículo en acceso abierto bajo términos de Licencia Creative Commons Atribución 4.0 Internacional. (http://creativecommons.org/licenses/by/4.0/)

ORCID iDs

Jorge Luis Vélez Páez

Santiago Xavier Aguayo Moscoso

Christian Leonardo Mora Coello

Wilson Daniel Álava Muñoz

María José Proaño Constante

Milagros Esthela Heredia Toledo
(1) https: / / orcid.org/0000-0002-6956-4475 (1) https: / / orcid.org/0000-0003-4919-5497

(1) https: / / orcid.org/0000-0002-3032-0537 (1) https: / / orcid.org/0000-0002-6114-6914 (1) https: / / orcid.org/0000-0002-5241-3099 (1) https: / / orcid.org/0000-0001-9765-3144 